\title{
ChemComm
}

\section{Alkynyl monosaccharide analogues as a tool for evaluating Golgi glycosylation efficiency: application to Congenital Disorders of Glycosylation (CDG) $\dagger$}

Received 2nd August 2013, Accepted 5th October 2013

DOI: $10.1039 / c 3 c c 45914 d$

\author{
Jorick Vanbeselaere, ${ }^{\text {ab }}$ Dorothée Vicogne, ${ }^{\text {ab }}$ Gert Matthijs, ${ }^{c}$ Christophe Biot, ${ }^{\text {ab }}$ \\ François Foulquier‡*ab and Yann Guerardelł $\star^{* a b}$
}

www.rsc.org/chemcomm

The visualization of Golgi glycosylation defects in patients' cells with Congenital Disorders of Glycosylation (CDG) is challenging and necessitates the use of cumbersome glycan analysis methods that are barely adapted to clinical research. We show here that metabolic labelling of patient cells with alkyne-tagged sialic-acid (SiaNAl) enables an easy and reliable readout assay for the detection of CDG occurrence. It also provides valuable clues regarding the pathological processes by assessing the distribution of sialic acid analogues within the cells.

The Congenital Disorders of Glycosylation (CDG) are a group of rare human genetic disorders characterized by defects in glycoconjugates that dramatically illustrate the fundamental involvement of glycosylation in the intricate design of life. In the past decade, about 60 different types of CDG have been defined genetically and most of the time, the defects impair the biosynthesis, addition (CDG-I) and/or remodelling of $N$-glycans (CDG-II). ${ }^{1}$ CDG-I disorders are characterized by reduced occupation of glycosylation sites on their newly synthesized glycoproteins while CDG-II disorders lead to the formation of abnormal glycan structures..$^{2-4}$ Different methods, based on HPLC and mass spectrometry (MS), have been applied and developed in the field of CDG to identify and quantify $\mathrm{N} / \mathrm{O}$-glycosylation deficiencies. ${ }^{5,6}$ While being extremely powerful, the sensitivity is often related to the amount of glycoproteins in a sample. Our goal was to develop an easy method applicable to non-specialized glycobiology laboratories to evaluate the Golgi glycosylation efficiency in mammalian living cells. To achieve this objective, we have evaluated the use of metabolic incorporation of

\footnotetext{
${ }^{a}$ Université Lille1, UGSF, F-59650 Villeneuve d'Ascq, France.

E-mail: yann.guerardel@univ-lille1.fr, francois.foulquier@univ-lille1.fr;

Fax: 3303204365 55; Tel: 330320434883

${ }^{b}$ CNRS, UMR 8576, F-59650 Villeneuve d'Ascq, France

${ }^{c}$ Laboratory for Molecular Diagnostics, Center for Human Genetics,

University of Leuven, B-3000 Leuven, Belgium

† Electronic supplementary information (ESI) available: Details of methods together with supporting figures and a list of abbreviations. See DOI: 10.1039/ c3cc45914d

‡ These authors contributed equally.
}

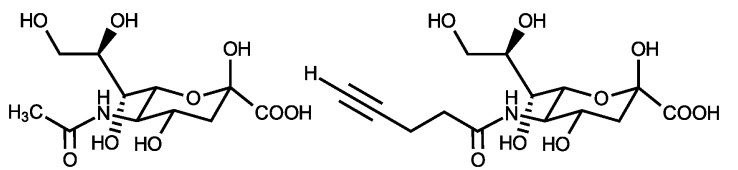

Fig. 1 Chemical structures of (left) $5-N$-acetyl- $\beta$-neuraminic acid (Neu5Ac) and (right) $N$-5-pentynoyl- $\beta$-neuraminic acid (SiaNAl).

unnatural monosaccharides into CDG fibroblasts, adapting the ligand-accelerated CuAAC using BTTAA and the alkynetagged sialic acid (SiaNAl). ${ }^{7,8}$ Although azido chemical reporter groups have been developed with success over a wide range of organisms, ${ }^{9,10}$ the SiaNal strategy has never been used in a human pathological context. Noteworthily, the use of azido-analogues to typify lysosomal disorders in Nemann-Pick disease was described in a recent report. ${ }^{11}$

In this context, we have synthesized alkyne-tagged sialic acid (SiaNAl) (Fig. 1) to metabolically incorporate into glycoconjugates and visualized it by the use of azido-functionalized fluorophores. This strategy was applied to cells from healthy individuals, CDG-I and CDG-II patient cells, and led us to identify and quantify the glycosylation defects in the different CDG patient cells. Fibroblasts from healthy individuals were metabolically labelled for periods ranging from 6 to $48 \mathrm{~h}$ to allow the incorporation of the alkyne-tagged sialic acid into newly synthesized glycoconjugates. After fixation, cells were permeabilized and labelled with an azido-545 fluorescent probe to visualize the pool of labelled sialylated glycoconjugates. As shown in Fig. S1 (ESI $\dagger$ ) we observed distinctive staining patterns suggesting differential subcellular localization of labelled sialylated glycoconjugates.

The alkyne-tagged sialic acid, irrespective of the incubation time, was clearly detected in unpunctuated regions as well as associated with vesicular structures localized throughout the cytoplasm (Fig. S1, ESI $\dagger$ ). After $24 \mathrm{~h}$ of labelling, the alkynetagged sialic acid, presumably bound to glycoproteins and glycolipids, was additionally observed at the cell surface, likely due to the targeting of labelled glycoproteins to the plasma membrane. To further delineate the subcellular localization of the different pools of labelled sialylated glycoconjugates, 

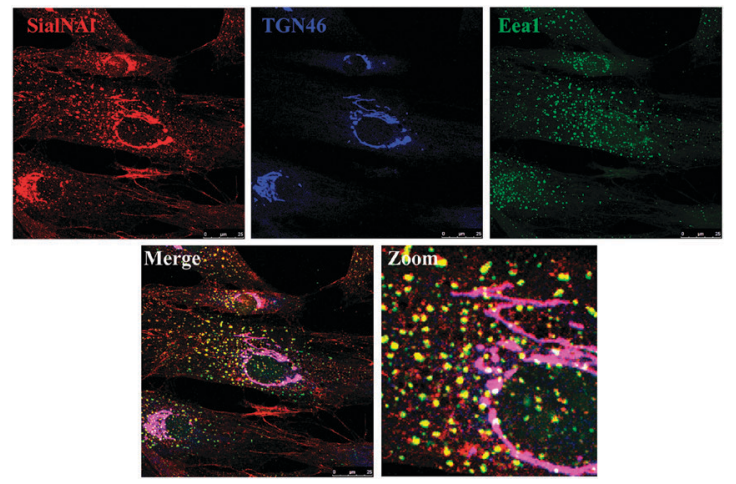

Fig. 2 Fibroblasts from healthy individuals were metabolically labelled with $500 \mu \mathrm{M}$ of SiaNAl and stained with azido fluorescent probes (sialic acid into glycoconjugates in red) or antibodies against a late Golgi marker (TGN46 in blue) and an early endosomal marker (EEA1 in green).

we performed immunofluorescence staining using organellespecific antibodies. TGN46 is a specific marker from the TGN compartment whereas EEA1 predominantly resides within early endosomes. As shown in Fig. 2, two pools of labelled sialylated glycoconjugates can be differentiated, one perfectly localized in the Golgi compartment and another one localized in early endosomes. Staining using different fixing methods further suggested that sialic acid analogues are linked to glycoconjugates in the identified compartments (Fig. S2, ESI $\dagger$ ).

We then compared the incorporation efficiency on a comprehensive panel of fibroblasts isolated from known CDG-I and II patients with fibroblasts from a healthy individual. Known defects included deficiencies in glycosyltransferases or monosaccharide processing enzymes (PMM2 and ALG11), Golgi transport proteins (COG7, COG5), Golgi transporters (ATP6V0A2, TMEM165), and an uncharacterized CDG-II patient presenting severe Golgi glycosylation deficiencies. Overall, a sharp decrease, depending on the molecular defects, of the fluorescence intensity associated to the pool of labelled glycoconjugates co-localizing with TGN46 was observed compared to control cells (Fig. 3 and Fig. S3, ESI $\dagger$ ).

Almost no fluorescence associated to alkyne-tagged bound sialic acid in the perinuclear Golgi region could be detected in CDG patients with COG7 defect, ATP6V0A2 defect and in the uncharacterized CDG-IIx patient (observed a decrease of 98\%, $93 \%$ and $74 \%$, respectively, compared to control). In contrast, only a slight decrease was seen in milder forms of CDG patients with defects in TMEM165, MAN1B1 and COG5 (11\%, 34\% and $44 \%$, respectively, compared to controls). Besides these defects, it was possible that abnormal incorporation of alkyne-tagged sialic acid could be caused by a sub-optimal occupation of glycosylation sites in glycoproteins, as seen in the CDG-I patients with defects in PMM2 and ALG11. Both types also presented an intermediate reduction level in the incorporation of sialic acid into glycoconjugates (56\% and 66\%, respectively, compared to control). The observed decreases are directly proportional to the severity of the known defects but not to the type of CDG. Nonetheless, it is noteworthy that type II CDG defects exhibited lower fluorescence intensity on average than type I.
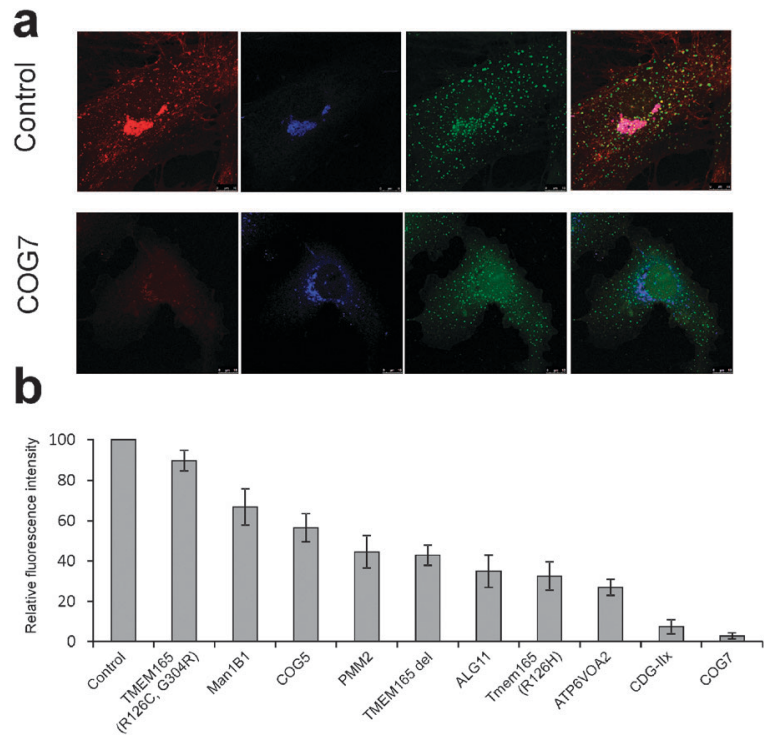

Fig. 3 Differential incorporation of tagged sialic acid into CDG deficient patients (see all panels in Fig. S2, ESIt). (a) Fibroblasts from healthy individuals and COG-7 deficient patients were metabolically labeled as in Fig. 2. (b) The associated fluorescence to the alkyne tagged sialic acid was quantified. For each cell line, 100 cells were quantified and the results are an average of two independent experiments. The errors bars represent the SEM.

Aware that the fluorescent quantification could be misrepresented due to the immunofluorescent protocol, we quantified the SiaNAl content of all carbohydrate-containing fractions from two CDG patients presenting very different patterns of alkyne-tagged sialic acid incorporation (PMM2 and COG7) and control fibroblasts. We purified glycoprotein-, glycolipid- and cytosolic free sialic acid-containing fractions from each cell type and quantified their contents in natural (Neu5Ac and Neu5Gc) and tagged (SiaNAl) sialic acids by reverse phase fluorescence HPLC. ${ }^{12}$ In control fibroblasts cultured with medium that was not supplemented with SiaNAl, all three fractions contained about $95 \%$ of Neu5Ac and 5\% of Neu5Gc (data not shown). Although only present as traces in human cells, significant quantities of Neu5Gc can be identified when grown in culture medium supplemented by BSA, likely due to the intake of bovine Neu5Gc that competes with endogenous Neu5Ac. ${ }^{13}$ When supplemented with SiaNAl, control fibroblasts contained 86 to $91 \%$ Neu5Ac, 4 to $6 \%$ Neu5Gc and 3 to $7 \%$ SiaNAl (data not shown).

As shown in Fig. 4a, the total amount of SiaNAl incorporated into CDG deficient cells was very similar to the one observed in control cells, establishing that the differences in incorporation into glycoconjugates observed by microscopy were not the result of the modification of sialic acid uptake but of intracellular trafficking and/or sialic acid utilization. Strikingly, and in accordance with the observed loss of Golgi fluorescence by microscopy, the incorporation of SiaNAl into glycoproteins dropped by $33 \%$ in PMM2 deficient CDG cells and by $66 \%$ in COG7 deficient CDG cells (Fig. 4b). Reciprocally, exogenous free SiaNAl strongly accumulated into the soluble cytosolic fraction of COG7 deficient CDG cells. Interestingly, no differences in the quantification of SiaNAl incorporated into glycolipids were observed. 
a

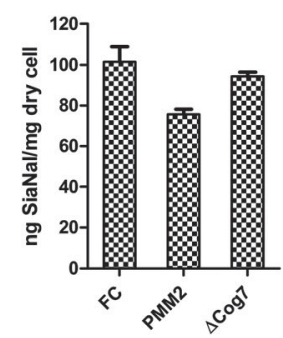

b

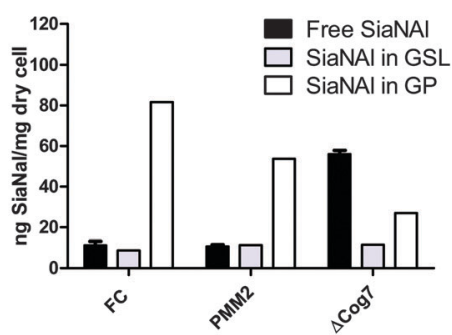

Fig. 4 Quantification by HPLC of the alkyne tagged sialic acid incorporation into glycoconjgates in deficient CDG patient cells versus control cells (FC). (a) Quantification of SiaNAl from total cells and (b) quantification of SiaNAl according to origin: free SiaNAl, bound to glycosphyngolipid fraction (GSL) or glycoprotein fraction (GP). Values are averaged from three experiments. The error bars represent the SEM.

For this study, we took advantage of a large collection of characterized CDG-I and CDG-II cell lines to establish that alkyne-tagged modified sialic acid can be used to easily assess the glycosylation defects in cultured cells from patients. Indeed, all CDG-II deficient cells were shown to express drastically lower quantities of newly synthesized glycoproteins than control cells. Defects in the Golgi retrograde trafficking as well as in Golgi pH and $\mathrm{Ca}^{2+}$ homeostasis lead to Golgi glycosylation abnormalities that involve all types of glycosylate substrates. ${ }^{14,15}$ Convincingly, our findings confirmed the severity of sialylation deficiency observed by mass spectrometry serum profiling in a much simpler and reliable way. It is particularly true for type II CDGs such as COG7 deficiencies for which the $\mathrm{N}$-glycome analysis is not related to the severity of the defect. ${ }^{16}$ Subcellular quantification of sialic acids by HPLC further established that this phenomenon may be the result of different processes. For PMM2 deficient CDG cells, the reduction of overall sialylation may originate from the combined effects of a reduced uptake of sialic acid from the medium and a reduced incorporation into glycoproteins. At the opposite, COG7 deficient patient cells did not exhibit any reduction in the sialic acid uptake but an incapacity to transfer it into their glycoproteins, leading to the accumulation of large quantities of unbound sialic acid in the cytoplasm.

We used hydroxyl-free sialic acid analogues rather than peracetylated ones in order to minimize non-specific crossing of internal membranes that may result in abnormal localization of sialic acids. In accordance with the reported data we showed that exogenous alkyne-tagged sialic acid (SiaNAl) could be efficiently incorporated into cells via newly synthesized Golgi glycoproteins. ${ }^{17}$ In our assay, two pools of alkyne-tagged sialic acids were clearly observed: one in TGN46 positive structures likely bound to Golgi glycoproteins and one in EEA1 positive

early endosomal structures. The subcellular localization of this pool suggests a plasma membrane recycling of SiaNAl tagged glycoproteins. Although the entry mechanisms of alkyne tagged sialic acid are not entirely solved, one could imagine that sialic acids enter into the cell by endocytosis. ${ }^{13}$ This tool could be used to follow the efficacy of the endocytic pathway which deficiencies may also lead to Golgi defects.

To conclude, we propose a novel chemical method based on the estimation of the Golgi glycosylation efficiency in patient cells with suspected glycosylation defects. This strategy may not only lead to the identification or confirmation of patients with glycosylation defects but is also of interest to exclude CDG-II patients presenting an abnormal transferrin isoelectrofocalisation (TIEF) profile due to secondary causes of hyposialylation as for patients with severe liver pathology and hemolytic uremic syndrome. We anticipate that this method will facilitate further biochemical and cellular experiments in the near future and speed up the diagnosis of patients with glycosylation defects.

\section{Notes and references}

1 T. Hennet, Biochim. Biophys. Acta, 2012, 1820, 1306-1317.

2 H. H. Freeze, Curr. Mol. Med., 2007, 7, 389-396.

3 F. Foulquier, Biochim. Biophys. Acta, 2009, 1792, 896-902.

4 M. Theodore and E. Morava, Curr. Opin. Pediatr., 2011, 23, 581-587.

5 R. K. T. Kam, T. C. W. Poon, H. L. Y. Chan, N. Wong, A. Y. Hui and J. J. Y. Sung, Clin. Chem., 2007, 53, 1254-1263.

6 S. Wopereis, S. Grünewald, K. M. L. C. Huijben, E. Morava, R. Mollicone, B. G. M. van Engelen, D. J. Lefeber and R. A. Wevers, Clin. Chem., 2007, 53, 180-187.

7 C. Besanceney-Webler, H. Jiang, T. Zheng, L. Feng, D. Soriano del Amo, W. Wang, L. M. Klivansky, F. L. Marlow, Y. Liu and P. Wu, Angew. Chem., Int. Ed., 2011, 50, 8051-8056.

8 E. M. Sletten and C. R. Bertozzi, Acc. Chem. Res., 2011, 44, 666-676.

9 S. T. Laughlin, J. M. Baskin, S. L. Amacher and C. R. Bertozzi, Science, 2008, 320, 664-667.

10 A. Dumont, A. Malleron, M. Awwad, S. Dukan and B. Vauzeilles, Angew. Chem., Int. Ed., 2012, 51, 3143-3146.

11 N. E. Mbua, H. Flanagan-Steet, S. Johnson, M. A. Wolfert, G.-J. Boons and R. Steet, Proc. Natl. Acad. Sci. U. S. A., 2013, 110, 10207-10212.

12 J. Vanbeselaere, L.-Y. Chang, A. Harduin-Lepers, E. Fabre, N. Yamakawa, C. Slomianny, C. Biot, K.-H. Khoo and Y. Guerardel, J. Proteome Res., 2012, 11, 2164-2177.

13 M. Bardor, D. H. Nguyen, S. Diaz and A. Varki, J. Biol. Chem., 2005, 280, 4228-4237.

14 U. Kornak, E. Reynders, A. Dimopoulou, J. van Reeuwijk, B. Fischer, A. Rajab, B. Budde, P. Nürnberg, F. Foulquier, ARCL Debré-type Study Group, D. Lefeber, Z. Urban, S. Gruenewald, W. Annaert, H. G. Brunner, H. van Bokhoven, R. Wevers, E. Morava, G. Matthijs, L. Van Maldergem and S. Mundlos, Nat. Genet., 2008, 40, 32-34.

15 F. Foulquier, M. Amyere, J. Jaeken, R. Zeevaert, E. Schollen, V. Race, R. Bammens, W. Morelle, C. Rosnoblet, D. Legrand, D. Demaegd, N. Buist, D. Cheillan, N. Guffon, P. Morsomme, W. Annaert, H. H. Freeze, E. Van Schaftingen, M. Vikkula and G. Matthijs, Am. J. Hum. Genet., 2012, 91, 15-26.

16 M. Guillard, E. Morava, F. L. van Delft, R. Hague, C. Korner, M. Adamowicz, R. A. Wevers and D. J. Lefeber, Clin. Chem., 2011, 57, 593-602.

17 S. J. Luchansky, S. Goon and C. R. Bertozzi, ChemBioChem, 2004, 5, 371-374. 